\title{
TRANSPORTES AÉREOS NACIONALES (TANS) Una Empresa Estratégica para el Desarrollo
}

\author{
ALF. FIDEL CASTRO HERRERA Et. al (*)
}

\section{PRESENTACIÓN}

Los profundos cambios ocurridos en el ámbito mundial producto de la era de la globalización y de los adelantos científicotécnicos, más acelerados, han provocado que la totalidad de instituciones u organizaciones hayan tenido que variar sus funciones y estrategias como medida de adaptación, para así poder afrontar con posibilidades en este nuevo mundo caracterizado por la alta competencia y la diversidad de servicios.

El Perú no ha estado exento a este nuevo panorama, es más, ha respondido a ello como todos los países de la región (en su respectivo nivel); como resultado de esos cambios se percibe en la actualidad que tanto las instituciones públicas como las privadas han variado su accionar de acuerdo a las exigencias que se presentan hoy en día. En nuestro medio, la importancia de esos cambios se vuelve más acucioso cuando se tratan de instituciones tradicionales y tutelares; es decir, de organizaciones arraigadas en el tiempo y éste es el caso de la empresa aérea TANS.

Transportes 'Aéreos Nacionales ha resurgido para responder perfectamente los nuevos retos -ante las nuevas perspectivas que se presentan como producto de los acontecimientos antes mencionado- ampliando positivamente sus funciones $\mathrm{e}$ ingresando, así, decididamente al campo de la actividad civil. En su nuevo rol esta empresa se encuentra desempeñando una función de apoyo y de colaboración con el desarrollo económico y el progreso social del país.

'Ante este nuevo rol, el país se ha visto beneficiado con la presencia contundente de una empresa con estructura militar en el terreno civil, propiciando una sana competencia y mejorando los servicios con actividades de ingeniería, transporte y otras que han unido y desarrollado zonas inaccesibles y lejanas de nuestro país. Siendo este aspecto sólo el inicio del rol y papel que jugará dicha institución en el futuro cercano, en el conjunto del Estado dándole una nueva dimensión al aspecto de la seguridad nacional, por un lado, y al bienestar general, por el otro. Estos dos últimos aspectos son dos grandes objetivos imprescindibles para el desarrollo de cualquier sociedad moderna.

La presencia de TANS en el mercado está activando la vida empresarial, mediante la competencia en igualdad de condiciones con el medio civil altamente eficiente, donde los beneficiados son los usuarios.

$\left(^{*}\right)$ Bachiller en Administración. 
TANS resurge ante la falta de una línea de bandera nacional y debido a los acontecimientos acaecidos en el pasado. Esta empresa, con una moderna organización, toma la responsabilidad de ser la representante nacional y con capitales íntegramente nacionales. Su participación en el mercado tan competitivo de la aviación comercial le permite visualizar nuevos escenarios y nuevos retos. Ante este nuevo reto TANS viene desempeñándose impecablemente, respondiendo eficientemente ante cualquier condición y manteniendo una sólida posición por ser una empresa nacional próspera.

TANS viene desarrollando el servicio de transporte de pasajeros y carga a nivel nacional como una empresa aerocomercial del país, ofreciendo un valor agregado el cual se refleja en la diferenciación con la competencia y, éstos son sus principios corporativos que utiliza para despeñarse en la actualidad y en el futuro, separado de las políticas y teniendo en claro el ambiente cambiante que se presenta.

En base a los principios y a la experiencia aeronáutica, tales como: Atención esmerada y oportuna, economía y seguridad (por su característica enteramente militar), y los nuevos como son: el de flexibilidad, tecnología y competitividad. TANS se mantiene y aspira a convertirse en la línea aerocomercial líder del país, con proyección internacional.

Así como TANS podrán surgir otras empresas de origen militar que colaborarán también con la economía generando un aumento y mejoramiento en la producción de servicios, movilizando así el aparato productivo nacional.

Además, se puede apreciar cómo la cohesión de ventajas provenientes de doctrinas militares unidas con las del conocimiento del ámbito competitivo actual se pueden unir para formar organizaciones poderosas e integrales que pueden desempeñarse satisfactoriamente en cualquier campo y esto se logra con una presencia disciplinada y castrense en nuestro medio actual, es decir, el conocimiento del ámbito militar.

Finalmente, se puede concluir con la afirmación que este tipo de empresas laboran eficaz y eficientemente desarrollando sus fuerzas para consolidar y mantener la seguridad integral de la nación; a su vez tiene la capacidad para intervenir en el ámbito civil colaborando con el desarrollo económico basado en las capacidades y conocimientos que poseen, lo contrario no es cierto.

\section{CONSTITUCIÓN DE LA EMPRESA}

Por Decreto Supremo $N^{0} 17$ del 05/12/93 se crea TANS, por D.S. $N^{\circ} 013$ del 08/11/96 se regulariza TANS quedando inscrita en al As. 1 Folio 93, partida XIV, del tomo 12, del Registro Mercantil de Loreto.

Misión.- Brindar un servicio de transporte de pasajeros y carga a nivel nacional e internacional, eficiente, seguro, rentable, de alta calidad, con personal y material que permitan una proyección internacional.

Visión.- Convertirse en la línea aérea líder en el Perú con proyección internacional. Ocupar una gran parte del campo aeronáutico basándose en el progreso regular, siguiendo los principios de vocación de servicio y apoyo al desarrollo sociocultural del país con proyección hacia el campo internacional. Todo esto adecuándose a las políticas y cambios que surgieran en este mundo globalizado. 
Principios Corporativos (Definidos por el Presidente del Directorio de TANS TTG Fernando Suito Hermosilla)

- Lograr la eficiencia en proveer un servicio de calidad en base a la experiencia aeronáutica.

- Mantener la política de otorgar un buen servicio mediante una puntualidad adecuada.

-Atención al pasajero que no debe dejar de lado en ningún momento por ser de suma importancia para la organización.

- Otorgarle la máxima importancia en la seguridad de vuelos.

Tener un personal perfectamente concientizado con dichos principios implica desarrollar una buena selección de personal calificado que a la postre permitirá contar con una empresa aérea eficaz, segura y rentable.

\section{Problemas de la empresa}

- Falta una completa información, por parte del personal encargado, que dificulta la comunicación con posibles usuarios del servicio.

- El avión Fokker F28 con que cuenta TANS no ofrece las comodidades necesarias para un servicio aéreo adecuado (no como las de un avión BOEING 737), por lo que resulta incómodo, hasta cierto punto, viajar en él.

- La atención al cliente es menor que el de la principal competencia (Aerocontinente) que despliega mayor personal, con información adecuada y trato al pasajero con mayor cordialidad y preocupación.
- La publicidad y las promociones son sobrepasadas por la competencia la cual despliega una serie de promociones regulares acaparando así un gran porcentaje de usuarios del transporte aéreo.

- La competencia en su etapa de crecimiento ya ofrece vuelos internacionales con lo que está acaparando un mayor número de pasajeros, cosa que debería seguir TANS como la utilización del Benchmarking.

\section{PRINCIPIOS CORPORATIVOS}

Puntualidad.-Cumplir los compromisos y obligaciones pactados constantemente, con cualquier persona, en el tiempo acordado. Permite dar confianza en que la organización no falta a sus clientes, por lo que espera lo mismo de sus proveedores, a fin de mantener este valor.

Seguridad.- Consiste en inspirar confianza en todos los aspectos: prevención de accidentes, manejo del equipaje, instalaciones, equipos, póliza de seguros, etc. Para que todas las personas que entren en contacto con la organización se sientan protegidos ante cualquier eventualidad.

Economía.-Distribución adecuada de los recursos para obtener el máximo provecho de ellos, tanto de la empresa en beneficio de los clientes como de los proveedores hacia la empresa.

Comodidad.- Por ser del rubro de servicios, es necesario buscar el máximo confort de los clientes al momento de disfrutar del servicio. También para los colaboradores, a fin de generar un clima agradable en el trabajo, y alcanzar la eficiencia.

Competitividad.-La calidad de la empresa le debe dar la capacidad de competir en el mercado para lograr que los usuarios puedan 
elegir entre las posibilidades existentes y así alcanzar niveles de calidad y excelencia.

Rentabilidad.- La empresa debe maximizar sus utilidades operando con eficiencia para producir ganancias y satisfacer a sus accionistas, cumplir con sus trabajadores, con el estado (pago de impuestos) y con sus proveedores.

Calidad de servicio.- En todo momento y lugar dar al cliente, y a la sociedad, la posibilidad de acceder o satisfacer sus

\section{Empresa Aérea de Transportes TANS}

\section{DEFINICIÓNDECULTURA}

Fundación.- Por Decreto Supremo $N^{\circ} 017$ del $05 / 12 / 63$ se crea TANS, por D.S. $N^{\circ}$ 013 del 08/11/66 se regulariza TANS, quedando inscrita en el As. 1 Folio 93, partida XIV, del tomo 12 , del registro mercantil de Loreto.

\begin{tabular}{|l|c|c|c|c|c|c|c|}
\hline & Sociedad & Estado & Clientes & Proveedores & Colaboradores & Accionistas & Competencia \\
\hline \hline Puntualidad & $\mathrm{X}$ & $\mathrm{X}$ & $\mathrm{X}$ & $\mathrm{X}$ & & & \\
\hline Seguridad & $\mathrm{X}$ & $\mathrm{X}$ & $\mathrm{X}$ & & $\mathrm{X}$ & $\mathrm{X}$ & \\
\hline Economía & & & $\mathrm{X}$ & $\mathrm{X}$ & & & \\
\hline Comodidad & & & $\mathrm{X}$ & & $\mathrm{X}$ & & \\
\hline Competitividad & & & & & & & $\mathrm{X}$ \\
\hline Rentabilidad & & & $\mathrm{X}$ & $\mathrm{X}$ & $\mathrm{X}$ & $\mathrm{X}$ & \\
\hline $\begin{array}{l}\text { Calidad de } \\
\text { Servicio }\end{array}$ & $\mathrm{X}$ & & $\mathrm{X}$ & & & & \\
\hline Responsabilidad & $\mathrm{X}$ & $\mathrm{X}$ & $\mathrm{X}$ & $\mathrm{X}$ & $\mathrm{X}$ & $\mathrm{X}$ & \\
\hline Tecnología & & & $\mathrm{X}$ & & $\mathrm{X}$ & & \\
\hline
\end{tabular}

necesidades con valores agregados que superen sus expectativas.

Responsabilidad.- Ante todos los grupos con quienes es necesario asumir las acciones realizadas, aceptando las fallas y atendiendo las consecuencias para corregir y mejorar.

Tecnología-Como herramienta para lograr la calidad, mejorando las condiciones de trabajo, comodidad, economía y seguridad.

\section{Crecimiento y Desarrollo}

A medida que se desarrolla la empresa de Aéreo Transportes TANS se abarcan varios aspectos fundamentales que no podemos dejar de lado, como por ejemplo:

- Un acuerdo mutuo de socialización de miembros: donde permita que la empresa compita con los más altas organismos nacionales. 
- Capacitación del personal: donde orgullosamente se presentan atención personalizada, calidad de servicio y excelencia que nos distingue.

\section{Niveles de Cultura}

\section{1) Artefactos}

Arquitectura en el Ambiente Físico

- $\quad$ Agencias más pequeñas y mobiliarios modernos que permiten mayor cantidad de agencias; expansión, además de innovación en los aviones que son el Boeing 707, 797, 727 con colores que indica dinamismos, juventud y una simbología patria: blanco y rojo.

\section{El Lenguaje}

- Atención personalizada del cliente.

- Los bauchers (atención del cliente en caja) deben estar interbasados en la atención al cliente máximo.

- El lenguaje corporal: sonreír, es la gran fortaleza que pretende compensar el tiempo empleado en esperar su turno.

- Lemas al respecto: "Volamos Uniendo al Perú".

\section{Tecnología de sus productos}

- Servicio descentralizado y $100 \%$ interconectado.

- Vuelo efectivo garantizado incondicionalmente por nuestra empresa TANS.

- Horario corrido de vuelos nacionales durante los 7 días de la semana.

- Rapidez, exactitud y claridad en todas las actividades relacionadas con los clientes.

\section{Las Creaciones Artísticas}

Atención de personal joven y agraciado con presencia ejecutiva.

- Uniforme de colores muy alegres.

- Avisos publicitarios.

\section{2) Valores}

TANS deberá buscar de manera permanente el crecimiento de su valor como empresa, en la que los clientes constituyen su razón de ser, dentro de una sana competencia con las demás líneas aéreas, pero manteniendo nuestra clara presencia institucional en la sociedad.

\section{Vocación de Servicio}

Atender siempre con cortesía, eficiencia y amabilidad al cliente.

\section{Calidad de Nuestro Trabajo}

Satisfacer la necesidad del cliente de manera oportuna y eficaz cumpliendo con el horario de vuelo establecido.

\section{Cooperación Plena}

Demostrada en la disposición para trabajar en equipo, recibir y proporcional informes claros y oportunos para encontrar la solución a los problemas que pueden presentarse.

\section{Adaptación al cambio}

Flexibilidad para adecuarse a situaciones nuevas o imprevistas.

\section{Creatividad}

Expresada en el aporte de nuevas rutas $y / o$ ideas que puedan ser aplicables para un mejor desempeño empresarial en el campo aeroespacial.

\section{Profesionalismo}

Realizando los vuelos con responsabilidad e iniciativa, aplicando los conocimientos adquiridos, las técnicas y experiencia para lograr resultados eficaces.

Honestidad

Descripción para actuar siempre con integridad y rectitud. 
Respeto a los demás

Escuchar, comprender y respetar los puntos de vista de otras personas ofreciendo un trato cortés y racional.

\section{Voluntad de diálogo}

Facilitando la información que otras personas requieren para un mejor desempeño en las labores cotidianas.

\section{Desprendimiento}

Llevando a cabo mediante la transmisión de nuestro conocimientos y experiencia a favor de nuestros compañeros de trabajo.

\section{Iniciativa}

Buscando continuamente nuevas y mejores formas de trabajo que requiere una mayor productividad y mejor servicio al cliente.

\section{3) Creencias}

- TANS, es leal con las demás instituciones y con sus compañeros.

- Su desempeño es siempre digno, respetando la verdad.

- Respeta y hace respetar las leyes y otros dispositivos legales que norman la actividad de la empresa.

- Se diferencia de la competencia por la mística de trabajo y el compromiso por hacer bien las cosas.

- Los intereses personales no deben entrar en conflicto con la empresa TANS.

- Se obtiene de negociar o hacer contrato alguno a nombre de la empresa con cualquier entidad de la cual formamos parte como accionistas o que pertenezcan a familiares directos.

- No discrimina a los clientes, proveedores, compañeros por su sexo, creencia religiosa, etc.

\section{ANÁLISIS INTERNO TANS}

\section{DIAGNÓSTICO INTERNO PCI}

\begin{tabular}{||c|c|c|c|c|c|c|c|c|c|}
\hline \multirow{2}{*}{$\begin{array}{c}\text { CAPACIDAD } \\
\text { DIRECTIVA }\end{array}$} & \multicolumn{2}{|c|}{ FORTALFZA } & \multicolumn{3}{c|}{ DEBILIDAD } & \multicolumn{3}{c|}{ IMPACTO } \\
\cline { 2 - 8 } & Alta & Media & Bajo & Alta & Media & Bajo & Alta & Media & Bajo \\
\hline \hline $\begin{array}{l}\text { 1. Flexibilidad de la } \\
\text { Organización }\end{array}$ & & & & & $\mathrm{X}$ & & & & $\mathrm{X}$ \\
\hline 2. Imagen Corporativa & & $\mathrm{X}$ & & & & & & $\mathrm{X}$ & \\
\hline $\begin{array}{l}\text { 3. Agresividad Para } \\
\text { Enfrentar a la } \\
\text { Competencia }\end{array}$ & & & & $\mathrm{X}$ & & & & & $\mathrm{X}$ \\
\hline $\begin{array}{l}\text { 4. Toma de Decisiones } \\
\text { por Parte de los } \\
\text { Empleados }\end{array}$ & & & & & & & & & \\
\hline $\begin{array}{l}\text { 5. Orientación } \\
\text { Empresarial }\end{array}$ & $\mathrm{X}$ & & & & & & & & \\
\hline
\end{tabular}




\section{DIAGNÓSTICO INTERNO PCI}

\begin{tabular}{|c|c|c|c|c|c|c|c|c|c|}
\hline \multirow{2}{*}{$\begin{array}{l}\text { CAPACIDAD } \\
\text { COMPETITIVA }\end{array}$} & \multicolumn{3}{|c|}{ FORTALEZA } & \multicolumn{3}{|c|}{ DEBILIDAD } & \multicolumn{3}{|c|}{ IMPACTO } \\
\hline & Alta & Media & Bajo & Alta & Media & Bajo & Alta & Media & Bajo \\
\hline $\begin{array}{l}\text { 1. Rutas a Nivel } \\
\text { Internacional }\end{array}$ & & & & & $X$ & & $X$ & & \\
\hline $\begin{array}{l}\text { 2. Bajo Costo de los } \\
\text { Boletos }\end{array}$ & & & & $\mathrm{X}$ & & & & $\mathrm{X}$ & \\
\hline $\begin{array}{l}\text { 3. Rutas a Nivel } \\
\text { Nacional }\end{array}$ & & $\mathrm{X}$ & & & & & & $\mathrm{X}$ & \\
\hline $\begin{array}{l}\text { 4. Calidad en el } \\
\text { Servicio }\end{array}$ & & $\mathrm{X}$ & & & & & $\mathrm{X}$ & & \\
\hline $\begin{array}{l}\text { 5. Seguridad del } \\
\text { Equipaje }\end{array}$ & & & & & $\mathrm{X}$ & & $X$ & & \\
\hline
\end{tabular}

DIAGNÓSTICO INTERNO PCI

\begin{tabular}{||c|c|c|c|c|c|c|c|c|c||}
\hline \multirow{2}{*}{$\begin{array}{c}\text { CAPACIDAD } \\
\text { COMPETITIVA }\end{array}$} & \multicolumn{3}{|c|}{ FORTALEZA } & \multicolumn{3}{c|}{ DEBILIDAD } & \multicolumn{3}{c||}{ IMPACTO } \\
\cline { 2 - 9 } & Alta & Media & Bajo & Alta & Media & Bajo & Alta & Media & Bajo \\
\hline $\begin{array}{l}\text { 1. Disponibilidad de } \\
\text { Capital }\end{array}$ & $\mathrm{X}$ & & & & & & & & \\
\hline 2. Baja Rentabilidad & & & & & $\mathrm{X}$ & & $\mathrm{X}$ & & $\mathrm{X}$ \\
\hline 3. Manejo de Caja & & & & & & $\mathrm{X}$ & & $\mathrm{X}$ & \\
\hline 4. Costo de Abandono & & $\mathrm{X}$ & & & & & $\mathrm{X}$ & & \\
\hline 5. Costo de \\
Salvamento
\end{tabular}




\section{DIAGNÓSTICO INTERNO PCI}

\begin{tabular}{|c|c|c|c|c|c|c|c|c|c|}
\hline \multirow{2}{*}{$\begin{array}{l}\text { CAPACIDAD } \\
\text { COMPETITIVA }\end{array}$} & \multicolumn{3}{|c|}{ FORTALEZA } & \multicolumn{3}{|c|}{ DEBILIDAD } & \multicolumn{3}{|c|}{ IMPACTO } \\
\hline & Alta & Media & Bajo & Alta & Media & Bajo & Alta & Media & Bajo \\
\hline $\begin{array}{l}\text { 1. Aeronaves } \\
\text { Modernas }\end{array}$ & $X$ & & & & & & $\mathrm{X}$ & & \\
\hline $\begin{array}{l}\text { 2. Valor Agregado al } \\
\text { Servicio }\end{array}$ & & & & & $\mathrm{X}$ & & & $\mathrm{X}$ & \\
\hline $\begin{array}{l}\text { 3. Tecnología de sus } \\
\text { Centros de cómputo }\end{array}$ & & $X$ & & & & & & $\mathrm{X}$ & \\
\hline $\begin{array}{l}\text { 4. Arquitectura del } \\
\text { Ambiente Físico }\end{array}$ & & $X$ & & & & & $\mathrm{X}$ & & \\
\hline $\begin{array}{l}\text { 5. Seguridad } \\
\text { Descentralizada }\end{array}$ & & & $\mathrm{X}$ & & & & & & $\mathrm{X}$ \\
\hline
\end{tabular}

\section{DIAGNÓSTICOINTERNOPCI}

\begin{tabular}{|c|c|c|c|c|c|c|c|c|c|}
\hline \multirow{2}{*}{$\begin{array}{l}\text { CAPACIDAD } \\
\text { COMPETITIVA }\end{array}$} & \multicolumn{3}{|c|}{ FORTALEZA } & \multicolumn{3}{|c|}{ DEBILIDAD } & \multicolumn{3}{|c|}{ IMPACTO } \\
\hline & Alta & Media & Bajo & Alta & Media & Bajo & Alta & Media & Bajo \\
\hline $\begin{array}{l}\text { 1. Profesionalismo de } \\
\text { sus Miembros }\end{array}$ & $\mathrm{X}$ & & & & & & $\mathrm{X}$ & & \\
\hline 2. Motivación & & $\mathrm{X}$ & & & & & & $\mathrm{X}$ & \\
\hline $\begin{array}{l}\text { 3. Calidad en el } \\
\text { Trabajo }\end{array}$ & & $\mathrm{X}$ & & & & & $\mathrm{X}$ & & \\
\hline $\begin{array}{l}\text { 4. Subordinación a los } \\
\text { Demás }\end{array}$ & & & & $X$ & & & & & $\mathrm{X}$ \\
\hline $\begin{array}{l}\text { 5. Atención } \\
\text { Personalizada }\end{array}$ & $\mathrm{X}$ & & & & & & $\mathrm{X}$ & & \\
\hline
\end{tabular}




\section{MATRIZ DE EVALUACIÓN DE FACTORES INTERNOS DE LA EMPRESA "TANS"}

\begin{tabular}{|c|c|c|c|}
\hline ACTOR INTERNO CLAVE & PESO & RANGO & $\begin{array}{c}\text { PESO } \\
\text { PROMEDIO }\end{array}$ \\
\hline \multicolumn{4}{|l|}{ FORTALEZAS } \\
\hline 1. Profesionalismo de sus Miembros & 0,08 & 4 & 0,32 \\
\hline 2. Aeronaves Modernas & 0,08 & 4 & 0,32 \\
\hline 3. Imagen Corporativa & 0,04 & 3 & 0,12 \\
\hline 4. Calidad en el Servicio & 0,05 & 4 & 0,20 \\
\hline 5. Orientación Empresarial & 0,04 & 3 & 0,12 \\
\hline 6. Motivación & 0,03 & 4 & 0,12 \\
\hline 7. Calidad del Trabajo & 0,05 & 3 & 0,15 \\
\hline 8. Atención Personalizada & 0,04 & 3 & 0,12 \\
\hline 9. Disponibilidad de Capital & 0,05 & 4 & 0,20 \\
\hline 10. Arquitectura del Ambiente Físico & 0,04 & 4 & 0,16 \\
\hline \multicolumn{4}{|l|}{ DEBILIDADES } \\
\hline 1. Subordinación a los Demás & 0,04 & 1 & 0,04 \\
\hline 2. Valor Agregado al Servicio & 0,05 & 1 & 0,05 \\
\hline 3. Baja Rentabilidad & 0,06 & 1 & 0,06 \\
\hline 4. Seguridad del Equipaje & 0,04 & 2 & 0,08 \\
\hline 5. Bajo costo de los Boletos & 0,06 & 1 & 0,06 \\
\hline 6. Ruta a Nivel Internacional & 0,07 & 1 & 0,07 \\
\hline 7. Agresividad para la Competencia & 0,06 & 1 & 0,06 \\
\hline 8. Toma de Decisiones de los Empleados & 0,04 & 2 & 0,05 \\
\hline 9. Flexibilidad de la Organización & 0,05 & 1 & 0,05 \\
\hline 10. Manejo de Caja & 0,03 & 1 & 0,03 \\
\hline \multicolumn{4}{|c|}{ TOTAL } \\
\hline
\end{tabular}

$\begin{array}{lll}\text { RANGO } & \text { FORTALEZA MAYOR } & 4 \\ & \text { FORTALEZA MENOR } & 3 \\ & \text { FORTALEZA MENOR } & 2 \\ & \text { DEBILIDAD MAYOR } & 1\end{array}$

\section{ANÁLISIS}

Como hemos podido observar, el valor final del cuadro matriz de evaluación de factores internos de la empresa TANS nos da un valor de $(2,41)$ que nos indica que la empresa está trabajando de manera regular; pero le falta un poco más de estrategias para alcanzar un nivel más competitivo. 
MATRIZ POAM

EMPRESA DE TRANSPORTES AÉREOS - TANS

\begin{tabular}{|c|c|c|c|c|c|c|c|c|c|}
\hline \multirow{2}{*}{ CAPACIDADCOMPETITIVA } & \multicolumn{3}{|c|}{ FORTALEZA } & \multicolumn{3}{|c|}{ DEBILIDAD } & \multicolumn{3}{|c|}{ IMPACTO } \\
\hline & A & $\mathrm{M}$ & $\mathrm{B}$ & $\mathrm{A}$ & $\mathrm{M}$ & B & A & $\mathrm{M}$ & $\mathrm{B}$ \\
\hline ECONÓMICAS & & & & & & & & & \\
\hline - TIENE UN PRESUPUESTO FIJO & & $\mathrm{X}$ & & & & & $\mathrm{X}$ & & \\
\hline $\begin{array}{l}\text { - DEPENDEDELA VARIACIÓN DEL } \\
\text { PRECIODE LOS COMBUSTIBLES }\end{array}$ & & & & $\mathrm{X}$ & & & $\mathrm{X}$ & & \\
\hline $\begin{array}{l}\text { - SUJETO A LA POLÍTICA ECONÓMICA DEL } \\
\text { PAÍS }\end{array}$ & & & $\mathrm{X}$ & & & & & $\mathrm{X}$ & \\
\hline POLÍTICAS & & & & & & & & & \\
\hline $\begin{array}{l}\text { - SUJETOS A REFORMAS DE TRATADOS } \\
\text { REGLAMENTOS DE LA OACI }\end{array}$ & & & & & $\mathrm{X}$ & & & & $\mathrm{x}$ \\
\hline $\begin{array}{l}\text { - SUJETO A CAMBIO DE GOBIERNO } \\
\text { (POLÍTICA DEL PAÍS) }\end{array}$ & & & $\mathrm{X}$ & & & & $\mathrm{X}$ & & \\
\hline SOCIALES & & & & & & & & 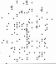 & \\
\hline $\begin{array}{l}\text { - IMPORTE DE SU ESTRUCTURA SOCIO- } \\
\text { ECONÓMICA }\end{array}$ & & & & & & & $\mathrm{X}$ & & \\
\hline $\begin{array}{l}\text { - INCREMENTO DE LAMIGRACIÓN A LAS } \\
\text { CIUDADES PRINCIPALES }\end{array}$ & & $\mathrm{x}$ & & & & & & $\mathrm{X}$ & \\
\hline - POLÍTICA SALARIAL & & $\mathrm{X}$ & & & & & $\mathrm{X}$ & & \\
\hline - NOHAY DISCRIMINACIÓN RACIAL & & & $\mathrm{x}$ & & & & & $\mathrm{x}$ & \\
\hline TECNOLÓGICAS & & & & & & & & & \\
\hline $\begin{array}{l}\text { - RECIBE APOYO DEMANTENIMIENTO DE } \\
\text { PERSONAL FAP }\end{array}$ & $\mathrm{X}$ & & & & & & $\bar{x}$ & & \\
\hline $\begin{array}{l}\text { - DIFICULTAD EN EL ACCESOA A LA } \\
\text { TECNOLOGÍA }\end{array}$ & & & & & $\mathrm{X}$ & & $\mathrm{X}$ & & \\
\hline $\begin{array}{l}\text { - GLOBALLZACIÓN ENEL ASPECTO } \\
\text { TECNOLOOGICO }\end{array}$ & & & & $\mathrm{X}$ & & & & $\mathrm{X}$ & \\
\hline $\begin{array}{l}\text { - RESISTENCIA A LOS CAMBIOS } \\
\text { TECNOLÓGICOS }\end{array}$ & & & & & $\mathrm{X}$ & & & & $\mathrm{X}$ \\
\hline $\begin{array}{l}\text { - BUENNIVEL TECNOLÓGICODELOS } \\
\text { INSUMOS UTILIZADOS }\end{array}$ & $\mathrm{X}$ & & & & & & $\mathrm{X}$ & & \\
\hline COMPETITIVOS & & & & & & & & 4 & \\
\hline $\begin{array}{l}\text { - ALTA COMPETENCIADEL TRANSPORTE } \\
\text { TERRESTRE }\end{array}$ & $\mathrm{X}$ & & & $\mathrm{X}$ & & & $\bar{X}$ & & \\
\hline - MERCADO AÉREO NACIONAL SATURADO & & & & & $\mathrm{X}$ & & $\mathrm{x}$ & & \\
\hline - NOEXISTEINVERSIÓNEXTRANJERA & $\mathrm{X}$ & & & & & & $\mathrm{X}$ & & \\
\hline $\begin{array}{l}\text { - POCACOMPETITIVIDADDEAEROLÍNEAS } \\
\text { NACIONALES }\end{array}$ & $\mathrm{X}$ & & & & & & $\mathrm{X}$ & & \\
\hline GEOGRÁFICAS & & & & & & & & & \\
\hline - LLEGA A LUGARES ALEJADOS DEL PAÍS & $\mathrm{X}$ & & & & & & $\mathrm{X}$ & & \\
\hline - FALTA DE INFRAESTRUCTURA PROPIA & & & & & $\mathrm{X}$ & & & $\mathrm{X}$ & \\
\hline
\end{tabular}




\begin{tabular}{|c|c|c|c|}
\hline FACTORES EXTERNOSCLAVES & PESO & RANGO & PESO PROM. \\
\hline \multicolumn{4}{|l|}{ OPORTUNIDADES } \\
\hline - LLEGA A PUNTOS ALEJADOS DEL PAÍS & 0,1 & 4 & 0,4 \\
\hline $\begin{array}{l}\text { - RECIBE APOYODEPERSONALY } \\
\text { MANTENIMIENTOFAP }\end{array}$ & 0,05 & 4 & 0,2 \\
\hline -RECIBEUN PRESUPUESTOFIJO & 0,08 & 4 & 0,32 \\
\hline $\begin{array}{l}\text { - SUJETA A REFORMAS Y TRATADOS DE } \\
\text { LA OACI }\end{array}$ & 0,01 & 3 & 0,03 \\
\hline $\begin{array}{l}\text {-BUENNIVEL TECNOLÓGICOENLOS } \\
\text { INSUMOS DE TRABAJO }\end{array}$ & 0,01 & 3 & 0,03 \\
\hline $\begin{array}{l}\text { - PRESENCIA DE LA CLASE MEDIA EN SU } \\
\text { ESTRUCTURA SOCIOECONÓMICA }\end{array}$ & 0,08 & 3 & 0,24 \\
\hline - MERCADO AÉREONACIONAL SATURADO & 0,02 & 3 & 0,06 \\
\hline $\begin{array}{l}\text {-LA GLOBALIZACIÓN EN EL ASPECTO } \\
\text { TECNOLÓGICO }\end{array}$ & 0,01 & 3 & 0,03 \\
\hline -NOHAY DISCRIMINACIÓN SOCIAL & 0,04 & 3 & 0,12 \\
\hline $\begin{array}{l}\text { POCACOMPETITIVIDAD DE AEREOLÍNEAS } \\
\text { NACIONALES }\end{array}$ & 0,1 & 4 & 0,4 \\
\hline AMENAZAS & 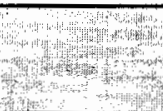 & (3) & (W) \\
\hline $\begin{array}{l}\text { - DIFICULTAD ENEL ACCESO A LA } \\
\text { TECNOLOGIA }\end{array}$ & 0,09 & 2 & 0,18 \\
\hline $\begin{array}{l}\text { - ALTA COMPETENCIA EN EL TRANSPORTE } \\
\text { TERRESTREY AÉREO }\end{array}$ & 0,1 & 1 & 0,1 \\
\hline $\begin{array}{l}\text { - DEPENDE DELA VARIACIÓN DEL PRECIO } \\
\text { DELCOMBUSTIBLE }\end{array}$ & 0,05 & 1 & 0,05 \\
\hline $\begin{array}{l}\text { - SUJETO A LA POLÍTICA ECONÓMICA DEL } \\
\text { PAÍS }\end{array}$ & 0,05 & 2 & 0,1 \\
\hline $\begin{array}{l}\text { - RESISTENCIA A LOS CAMBIOS } \\
\text { TECNOLÓGICOS }\end{array}$ & 0,02 & 2 & 0,04 \\
\hline $\begin{array}{l}\text { - INCREMENTO MIGRATORIO A CIUDADES } \\
\text { PRINCIPALES }\end{array}$ & 0,03 & 2 & 0,06 \\
\hline -INFRAESTRUCTURA COMPARATIVA & 0,05 & 1 & 0,05 \\
\hline $\begin{array}{l}\text {-SUJETOS A CAMBIOS DE GOBIERNO } \\
\text { (POLÍTICA NACIONAL) }\end{array}$ & 0,07 & 1 & 0,07 \\
\hline -NOEXISTEINVERSIÓNEXTRANJERA & 0,01 & 2 & 0,02 \\
\hline - POLÍTICA SALARIAL & 0,03 & 2 & 0,06 \\
\hline & 1 & tist & 2,56 \\
\hline
\end{tabular}

El resultado de to estudiado nos da como respuesta 2,56, esto quiere decir o nos indica que la empresa se encuentra y compite en un nivel intermedio; sus políticas internas son cumplidas de manera regular y los cambios externos que se pudieran producir no son significativos.

El entorno le es medianamente atractivo a TANS, en particular por las disponibilidad de llegar a puntos alejados y la poca competitividad de las demás líneas nacionales. 


\section{ANÁLISIS FODA "TANS"}

\section{FORTALEZAS}

1F Experiencia profesional de sus pilotos.

2F Acceso a aeródromos que no alcanzan otras compañias.

3F Entidad con apoyo del estado.

4F Emplea infraestructura propia deduciendo sus costos.

5F Existencia de pocos competidores en el mercado nacional.

$6 \mathrm{~F}$ Acceso a proveedores de suministros y servicios más económicos que la competencia.

7F Capacidad de brindar otros servicios que no posee la competencia.

8F Promoción de turismo interno.

9F Imagen orientada a las familias de sector medio.

10F Autonomía de su manejo, independiente de la fuerza aérea.

\section{DEBILIDADES}

1D Poca experiencia en el campo comercial.

2D Limitaciones en disponibilidad de aeronaves (propias).

3D Limitaciones para ampliar sus rutas al extranjero.

4D Escasa disponibilidad de recursos para realizar grandes inversiones (públicas e infraestructura).

5D Competidores agresivos.

6D Público no conforme con la calidad del servicio.

7D Protestas constantes de la competencia por el apoyo del estado.

8D Tecnología insuficiente en las aeronaves.

9D Alta rotación del personal.

10D Su subsistencia depende del mercado, el estado garantiza su permanencia.

\section{OPORTUNIDADES}

10 Puede llegar a puntos alejados del país.

20 Recibe apoyo total de personal y Mantto. FAP

30 Tiene asignado un presupuesto fijo.

40 Facilidad en la obtención de insumos de trabajo.

50 Disminución de la competitividad de aerolíneas nacionales.

60 No existencia de restricciones para el usuario.

70 Conocimiento y familiarización de las rutas aéreas.

80 Poder político actual con políticas de incentivo a la empresa privada.

90 Nivel socioeconómico bajo que atrae a los usuarios para utilizar servicios más baratos.

100 Apoyo del gobierno central al sector turismo.

\section{AMENAZAS}

1A Dificultad con el acceso a la tecnología

2A Fuerte presencia y competencia del medio de transporte terrestre.

3A Grandemente influenciada por las variaciones del precio del combustible.

4A Posible cambio de poder político.

5A Inmigración masiva a ciudades más importantes.

6A Aumento de la pobreza.

7A Renovación tecnológica incierta.

8A Desarrollo de la empresa sujeto a la política económica del país.

9A Menor inversión en capacitación y refaccionamiento en elámbito civil del personal en relación con el de competencia.

$10 \mathrm{~A}$ Visión corporativa y cultural organizacional más sólida por parte de la competencia. 
MATRIZ DE IMPACTO

FODA - PONDERADO

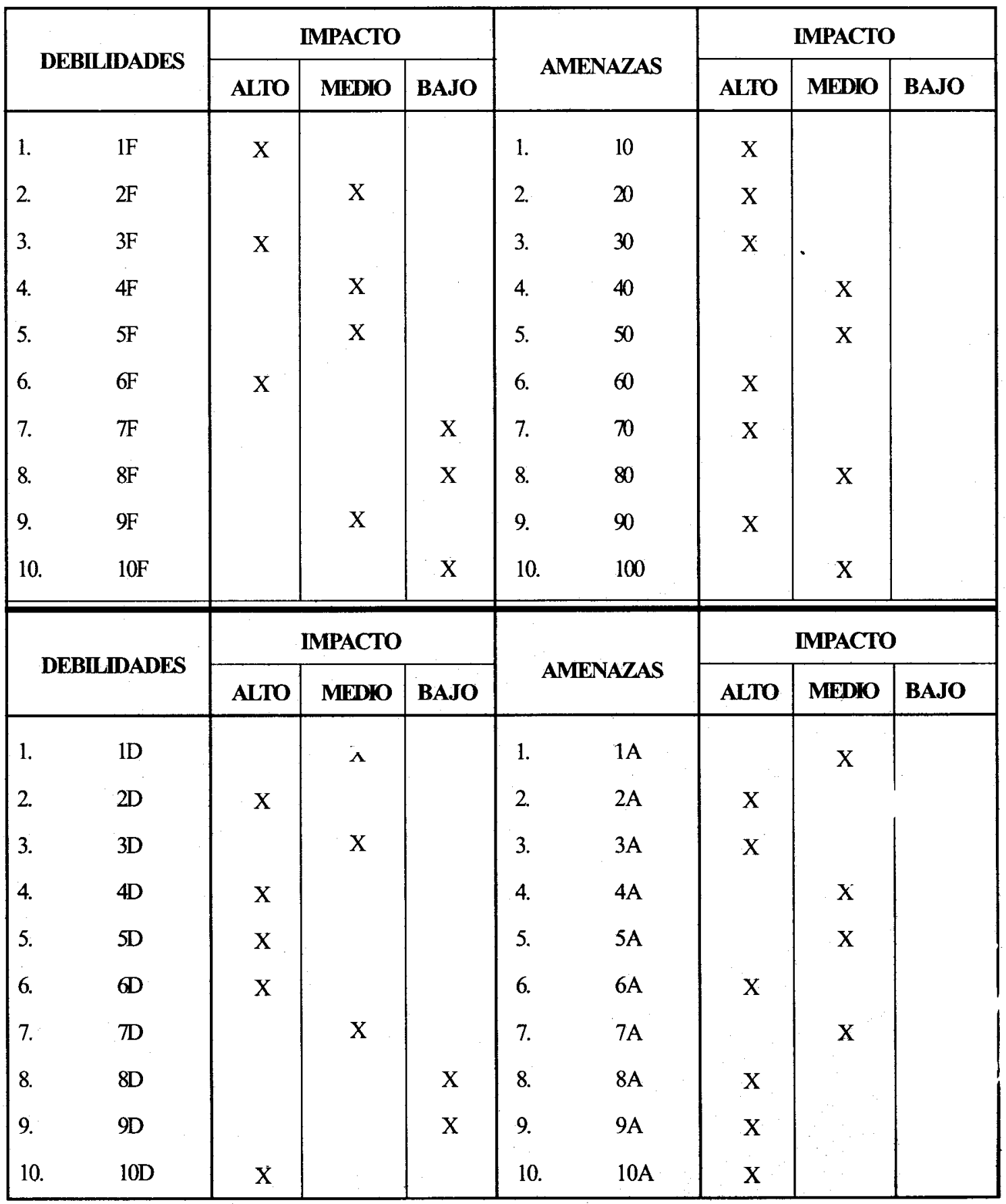




\section{ANÁLISIS FODA}

\begin{tabular}{|c|c|c|c|}
\hline & & OPORTUNIDADES & AMENAZAS \\
\hline & & $\begin{array}{ll}\text { 1. } & 1-0 \\
2 & 2-0 \\
\text { 3. } & 3-0 \\
\text { 4. } & 6-0 \\
\text { 5. } & 7-0\end{array}$ & $\begin{array}{ll}\text { 1. } & 1-0 \\
\text { 2. } & 2-0 \\
\text { 3. } & 3-0 \\
\text { 4. } & 6-0 \\
\text { 5. } & 7-0\end{array}$ \\
\hline & FORTALEZAS & ESTRATEGIAS & ESTRATEGIAS \\
\hline $\begin{array}{l}1 . \\
2 . \\
3 . \\
4 . \\
5 .\end{array}$ & $\begin{array}{l}1 \mathrm{~F} \\
3 \mathrm{~F} \\
6 \mathrm{~F} \\
2 \mathrm{~F}\left(^{*}\right) \\
9 \mathrm{~F}\left(^{*}\right)\end{array}$ & $\begin{array}{l}\text { Aprovechamiento de los } \\
\text { recursos para el incremento de } \\
\text { destinos (nacionales e } \\
\text { internacionales) } \\
\text { 3F-1.0 }\end{array}$ & $\begin{array}{l}\text { Utilización óptima de los } \\
\text { proveedores de suministros y } \\
\text { servicios para un desarrollo } \\
\text { próspero de la empresa. } \\
6 \mathrm{~F}-8 \mathrm{~A}\end{array}$ \\
\hline & DEBILIDADES & ESTRATEGIAS & ESTRATEGIAS \\
\hline $\begin{array}{l}1 . \\
2 . \\
3 . \\
4 . \\
5 .\end{array}$ & $\begin{array}{l}2 \mathrm{D} \\
4 \mathrm{D} \\
5 \mathrm{D} \\
6 \mathrm{D} \\
10 \mathrm{D}\end{array}$ & $\begin{array}{l}\text { Asignación mayor del presu- } \\
\text { puesto fijo destinado a mejorar } \\
\text { la calidad del servicio } \\
\qquad 30-6 \mathrm{D}\end{array}$ & $\begin{array}{l}\text { Destinar parte del presupuesto } \\
\text { asignado en el alquiler de } \\
\text { aeronaves para contrarrestar la } \\
\text { competencia en el ámbito } \\
\text { terrestre. } \\
\text { 2D - } 2 \mathrm{~A}\end{array}$ \\
\hline
\end{tabular}

(*) Fortalezas de Medio Impacto 